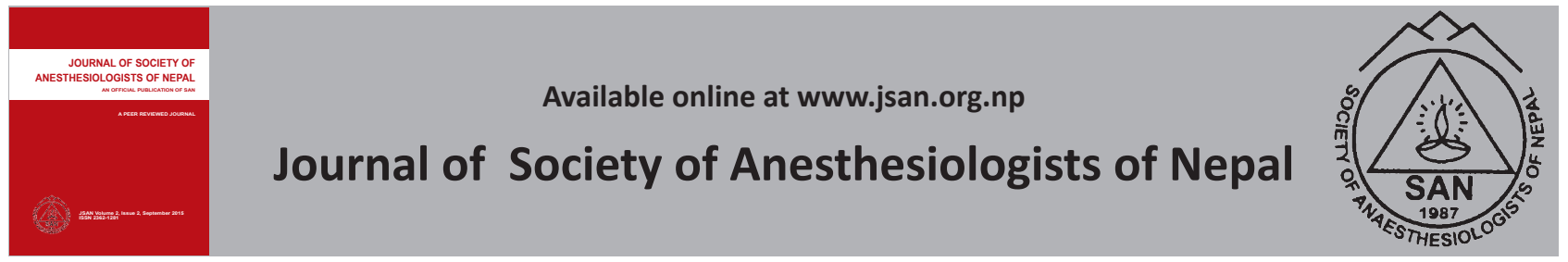

Original Article

\title{
Fiberoptic bronchoscope guided percutaneous dilatational tracheostomy in intensive care unit: a prospective observational study
}

\author{
Asfar Azimee, Taiyenjam Kennedy Singh \\ Shija Hospitals and Research institute, Meitei Langol, Lamphelpat, Imphal, Manipur 795004, India
}

A R I C L I F O
Received 03.03.2015
Accepted 07.09.2015
Published 10.09 .2015 C
Authors retain copyright
and grant the journal right
of first publication with the
work simultaneously licensed
under a Creative Commons
Attribution License that allows
others to share the work with
an acknowledgement of the
workss authorship and initial
publication in this journal.

\begin{abstract}
Background: The aim of the study was to evaluate the safety of fiberoptic bronchoscope guided percutaneous dilatational tracheostomy performed in the intensive care unit.

Methods: This was a prospective clinical study done on 30 critically ill patients in Intensive care unit. A puncture was made with $16 \mathrm{G}$ cannula at the second or third tracheal interspace which was confirmed by the fiberoptic bronchoscope. This was followed by insertion of guide wire through the cannula followed by insertion of the guiding catheter over the guide wire. The tract was enlarged with white single stage dilator to allow placement of a standard tracheostomy tube. The procedure was continuously monitored with the fiberoptic bronchoscope. Complications were noted during procedure and till patient's stay in Intensive care unit.
\end{abstract}

Results: The study included 18 (60\%) male and 12 (40\%) female patients. The mean age was $64.5 \pm 8$ years. Percutaneous dilatational tracheostomy was done early $(<10$ days) in 10 (33.3\%) patients and late (>10 days) in 20 (66.7\%) patients. Fentanyl was used for the procedure in all the patients and among them $8(26.6 \%)$ patients required injection rocuronium. Average procedure duration, from incision to suture for 30 patients was $12.6 \pm 2$ minutes. Indication for tracheostomy was weaning failure in 22 (73.3\%) patients and airway maintenance in 8 (26.6\%) patients. The mean duration patient remained on mechanical ventilation was 12.6 days and mean length of stay in critical care unit before shifting to ward was 7.6 days after tracheostomy. Acute postprocedure complications were transient bleed in four patients $(13.3 \%)$, two $(6.7 \%)$ had stomal bleeding, one (3\%) had tracheal mucosa laceration and another (3\%) had subcutaneous emphysema. No cases of stomal infection, pneumothorax, tracheal laceration, paratracheal insertion, pneumothorax and pneumomediastinum. There was no procedure-related mortality.

Conclusion: Fiberoptic bronchoscope guided percutaneous dilatational tracheostomy is safe and the method of choice for elective tracheostomy in the majority of intensive care patients.

Key words: adverse effects; bronchoscope; critically illness; intensive care unit; tracheostomy.

How to cite this article: Azimee A, Singh TK. Fiberoptic bronchoscope guided percutaneous dilatational tracheostomy in intensive care unit: a prospective observational study. JSAN 2015;2:52-55.

Corresponding author: Asfar Azimee, DA; Senior resident

Shija Hospitals and Research institute,

Meitei Langol, Lamphelpat,

Imphal, Manipur 795004, India

Telephone:+919310297021,

Email: azimee0071@gmail.com 


\section{Introduction}

Tracheostomy is generally done in Intensive Care Units (ICU) to facilitate weaning from mechanical ventilation, reduce anatomical dead space, avoid laryngeal injury and aid in management of tracheobronchial and pulmonary secretions. In selected ICU patients percutaneous dilatational tracheostomy technique has been shown to be safe ${ }^{1,2}$ and have fewer complications than surgical tracheostomy. 3,4,5,6,7 Surgical tracheostomy is usually reserved for patients with contraindications to percutaneous dilatational tracheostomy. What currently remains controversial is whether fiberoptic bronchoscope guided percutaneous dilatational tracheostomy has more or fewer complications as compared to the traditional surgical tracheostomy?

Therefore, to evaluate its safety we studied the outcomes of fiberoptic bronchoscope guided percutaneous dilatational tracheostomy, as performed by intensivist and anesthetists, on patients in our intensive care unit. All patients were carefully evaluated and followed up to assess the complications and adverse effects of percutaneous dilatational tracheostomy.

\section{Methods}

The study was a prospective study done from May 2014 to September 2014.The approval of hospital's ethical \& scientific committee was obtained. Written informed consent was taken from patient's first degree relative before subjecting the patient to study. All patients undergoing percutaneous dilatational tracheostomy were included in the study. Tracheostomy was performed for Weaning failure, which is defined as failure to pass a spontaneousbreathing trial or the need for re-intubation within 48 hours following extubation. ${ }^{8}$ The inclusion criteria was prolonged ventilation, need of airway protection against pulmonary aspiration (e.g. laryngeal incompetence due to critical illness, polyneuropathy, or bulbar dysfunction), prolonged need for intra-tracheal suction, upper airway obstruction, trauma or infection in oral cavity, pharynx or larynx, and the need to decrease sedation dose. Patients were excluded from the study if they had unstable cervical spine fractures, severe local infection of the anterior neck, coagulopathy ( platelet count is $<50 \times 10^{9}$, International normalised ratio $>1.5$, activated partial thromboplastin time $>50$ seconds or prolonged prothrombin time), local infection, high PEEP $\left(>8 \mathrm{cmH}_{2} \mathrm{O}\right)$ or $\mathrm{FiO}_{2}(>50 \%)$, difficult anatomy (e.g. morbid obesity, short thick neck, reduced neck extension, excessive goitre, tracheal deviation), proximity to extensive burns or surgical wounds, elevated intracranial pressure, previous radiotherapy to the neck that might need an emergency airway, children( $<15$ years) were excluded as there cartilages are soft. Indications for tracheostomy and mechanical ventilation in days before tracheostomy were recorded.

Pre-medication: Injection Fentanyl $(0.5-2 \mu \mathrm{g} / \mathrm{kg})$ was administered intravenously at the beginning of the procedure. If patient was still uncooperative Rocuronium $(0.6 \mathrm{mg} / \mathrm{kg})$ was given intravenously with $0.05 \mathrm{mg} / \mathrm{kg}$ of midazolam. $100 \%$ oxygen was applied to the patient for 10-15 min immediately prior to the procedure in order to prevent intraoperative hypoxia.

\section{Procedure}

Percutaneous dilatational tracheotomy technique was performed by Portex $^{\circledR}$ Percutaneous Dilation Tracheotomy Kit with Single Stage Dilator with Blue Line Ultra Tracheostomy Tube Introducer (Portex Ltd, Hythe, Kent, UK) with the insertion of a suitable sized tracheotomy tube. The procedure was performed by team of three or four specialists. One was intensivist and others were anesthesiologists having an experience of $>20$ percutaneous dilatational tracheostomy. To visualize the upper rings of the trachea, Fiberoptic bronchoscope (Olympus BF Type TE2) was used. Shoulder roll was placed and neck extended. The endotracheal tube was withdrawn until the cuff was at or just below the vocal cords. The anterior neck was prepared with povidone iodine and draped with sterile sheets. The skin overlying first and second tracheal rings was infiltrated subcutaneously with 2-3 $\mathrm{ml}$ of $2 \%$ Lignocaine with epinephrine $(1: 200,000)$, and a $1.5 \mathrm{~cm}$ transverse incision was made and blunt dissection was performed to expose the pre-tracheal fascia. The anterior trachea was palpated and the intended site was punctured with a $16 \mathrm{G}$ intravenous (IV) cannula in a posterio-caudal direction. The entry of the IV cannula in trachea was confirmed by aspiration of air into a saline filled syringe and also confirmed by bronchoscope. A guide wire was inserted through the cannula, and the cannula was withdrawn. This was followed by insertion of the guiding catheter over the guide wire. The tracheal opening was dilated over the guiding catheter using a single stage dilator until a stoma of sufficient size to accommodate the desired tracheostomy tube was created (Figure 1 and 2).

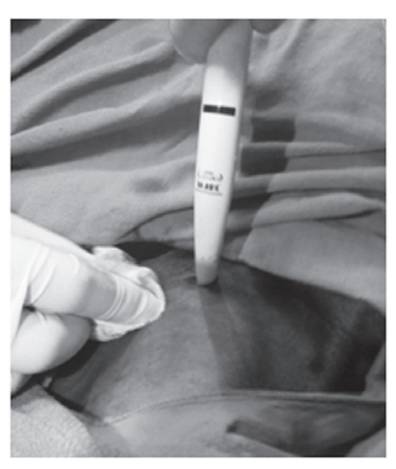

Figure 1

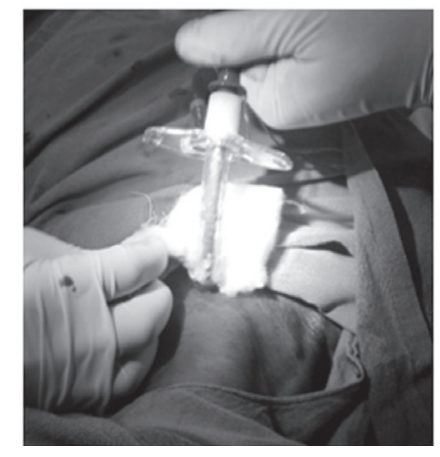

Figure 2
Figure 1: Dilatation of stoma with the use of single stage dilator

Figure 2: Insertion of tracheostomy tube into the trachea

Tracheotomy care included regular suctioning, humidified oxygen therapy, and regular wound care. Monitoring during and after the procedure included pulse rate, 
mean arterial blood pressure, oxygen saturation (using pulse oximetry), chest X-ray. All changes in the patients' clinical condition were carefully monitored i.e. changes in electrocardiography, blood pressure, heart rate, respiratory rate, fall in oxygen saturation, capnography. The complications that were potentially related to the procedure were noted during procedure and up to patient's stay in Intensive care unit e.g. cardiopulmonary arrest, conversion to surgical technique, major bleeding that caused hypotension, led to airway compromise, or required conversion to a surgical procedure to control it, pneumothorax/pneumomediastinum, subcutaneous emphysema, and/or misplacement or false passage into paratracheal tissues. Post-operative complications that were followed up after the procedure during stay in the intensive care unit included, tube obstruction or displacement, aspiration pneumonia, wound infection, haemorrhage; external or intra-tracheal, over dilatation of the stomal opening, and/or posterior tracheal wall lesion.

\section{Results}

Among the total 30 critically ill patients who underwent percutaneous dilatational tracheostomy in our intensive care unit $18(60 \%)$ were males and $12(40 \%)$ were females. The mean age was $64.5 \pm 8$ years.

Among the patients selected for the study, indication for mechanical ventilation was pulmonary infection in 12 patients, post abdominal surgery in 6 patients, road traffic accident in 6 patients, chronic obstructive pulmonary disease in 4 patients and central nervous system pathology in 2 patients. Indication for tracheostomy was weaning failure in 22 (73.3\%) and airway maintenance in $8(26.6 \%)$ patients. The mean duration patient remained on mechanical ventilation was 12.6 days and mean length of stay in critical care unit before shifting to ward was 7.6 days after tracheostomy.

Percutaneous dilatational tracheostomy was done early (<10 days) in 10 (33.3\%) patients and late (>10 days) in $20(66.7 \%)$ patients. In all the patient injection fentanyl was used for the procedure and among them $8(26.6 \%)$ patients required injection rocuronium. The mean procedure duration was $12.6 \pm 2$ minutes (from skin incision to suture time). Acute post-procedure complications were $(13.3 \%)$ transient bleed in four patients, one $(3 \%)$ patient had tracheal mucosa laceration and another one (3\%) had subcutaneous emphysema. Two patients had post-procedure in-hospital complication (6.0\%), the sole problem being stomal bleeding. No cases of stomal infection, pneumothorax or tracheal laceration or tracheal stenosis occurred and none of the patients desaturated during the procedure.

\section{Discussion}

The most common complications associated with percutaneous dilatational tracheostomy are haemorrhage, hypoxemia, loss of airway, cannula misplacement, airway injury, pneumothorax, surgical emphysema, damage to posterior tracheal wall and accidental decannulation in the immediate perioperative period. ${ }^{9}$ In a meta-analysis by Freeman et al, percutaneous dilatational tracheostomy was easier to perform, produced fewer overall postoperative complications, and had shorter operative times, less postoperative and perioperative bleeding, and fewer postoperative stomal infections. ${ }^{10}$ Though our study did not compare surgical tracheostomy with percutaneous, our results also suggest similar findings.

Based on available evidence from randomized controlled trials, 11, 12, 13, 14 there is no mortality benefit for early tracheostomy ( $\leq 10$ days) as compared to the late tracheostomy (>10days). However early tracheostomy was associated with more ventilator-free days, early weaning from mechanical ventilation and a shorter Intensive care unit length of stay. ${ }^{15}$ Therefore, the timing of tracheostomy is the prerogative of the intensivist, dictated by the patient's clinical status. Two third of our study population had undergone late tracheostomy.

Fibre optic bronchoscopy can be used to identify the point of needle insertion into the trachea and confirm correct guide-wire placement. ${ }^{16}$ The use of a bronchoscope may also facilitate teaching and supervision of inexperienced operators. Bronchoscopic guidance may potentially minimize the risk of complication(s), especially posterior tracheal wall injury. ${ }^{17,18}$ A post procedural bronchoscopy can also be performed to check tracheostomy position and for clot occlusion or cuff herniation. We used bronchoscopic guidance in all the patients. Bronchoscopic guidance facilitated positioning of endotracheal tube and helped in identifying the puncture site.

Percutaneous dilatational tracheostomy reduces the overall incidence of wound infection and may further reduce clinical relevant bleeding and mortality when compared with surgical tracheostomy performed in the operating theatre. ${ }^{6}$ Our study too, confirms the findings of earlier study. We attribute our low complication rates to our insertion procedure, in which percutaneous dilatational tracheostomy was performed in the intensive care unit under bronchoscope guidance by a team of experienced intensivist. In recent years, the placement of a tracheostomy has gained popularity as a means of facilitating the weaning of patients from the ventilator, as it reduces pulmonary dead space, provides access for the clearing of pulmonary secretions under various pathologic conditions, and improves the patient's comfort.

Percutaneous dilatational tracheostomy has a number of advantages including less procedure time and bleeding, decreased risk of perforation of the posterior wall of trachea and smaller skin incision. As earlier studies suggested, the safety of the procedure may be enhanced by the use of ultrasound. The incidence of bleeding which occurred during procedure can be further reduced by the use of ultrasound. The use of ultrasound in the procedure can 
be basis of future research. ${ }^{18}$ The safety of procedure has improved with prior screening of anterior neck anatomy as pre-percutaneous dilatational tracheostomy procedure preparation, ${ }^{20}$ whereas the real time ultrasound guided percutaneous dilatational tracheostomy being performed in patients with neurological damages has shown that the procedures were performed in relatively successful, safe and convenient manner. ${ }^{21}$ Our observations here only confirm the value of bronchoscopic guidance.

However, the results are applicable for patients aged $\geq$ 15 years, single centre study, small sample size were the limitations of the study.

\section{Conclusion}

We conclude that bronchoscopic guidance for percutaneous dilatational tracheostomy is the method of choice for elective tracheostomy in the majority of intensive care patients with a low rate of complications and mortality.

\section{Conflicts of interest: none}

\section{References}

1. Ambesh SP, Pandey CK, Srivastava S. Percutaneous tracheostomy with single dilation techniques. A prospective randomized comparison of Ciaglia Blue Rhino versus Griggs guide wire dilating forceps. Anesth Analg 2002;95:1739-45.

2. Diaz-Reganon G, Minambres E, Ruiz A. Safety and complications of percutaneous tracheostomy in a cohort of 800 mixed ICU patients. Anaesthesia 2008;63:1198-203

3. Friedman Y, Fildes J, Mizock B. Comparison of percutaneous and surgical Tracheostomies. Chest 1996;110:480-5.

4. De Leyn P, Bedert L, Delcroix M. Tracheotomy: clinical review and guidelines. Eur J Cardiothorac Surg 2007;32:412-21.

5. Antonelli M, Michetti V, Di Plama A. Percutaneous translaryngeal versus surgical tracheostomy: A randomized trial with 1-yr doubleblind follow-up. Crit Care Med 2005;33:1015-20.

6. Delaney A, Bagshaw SM, Nalos M. Percutaneous dilatational tracheostomy versus surgical tracheostomy in critically ill patients: a systematic review and meta-analysis. Crit Care 2006;10:R55.

7. Holdgaard HO, Pedersen J, Jensen RH, Outzen KE, Midtgaard T, Johansen LV et al. Percutaneous dilatational tracheostomy versus conventional surgical tracheostomy. Acta Anaesthesiol Scand 1998;42:545-50.

8. Heunks LMA, van der Hoeven JG. Clinical review: The $A B C$ of weaning failure - a structured approach. Critical Care 2010;14:245.

9. Durbin CG. Early Complications of Tracheostomy. Respir Care 2005;50:511-15.

10. Freeman $\mathrm{BD}$, Isabella K, Lin N. A meta-analysis of prospective trials comparing percutaneous and surgical tracheostomy in critically ill patients. Chest 2000;118:1412-8.

11. Barquist ES, Amortegui J, Hallal A, Giannotti G, Whinney R, Alzamel
$\mathrm{H}$ et al. Tracheostomy in ventilator dependent trauma patients: a prospective, randomized intention to- treat study. The Journal of Trauma 2006;60:91-7.

12. Adams JW, Hazard PB. A prospective, randomized, study comparing early percutaneous dilatational tracheotomy to prolonged Translaryngeal intubation (delayed tracheotomy) in critically ill medical patients. Critical Care Medicine 2004;32:1689-94.

13. Berardino M, Pallavicini FB. Early vs late tracheotomy for prevention of pneumonia in mechanically ventilated adult ICU patients: a randomized controlled trial. JAMA 2010;303:1483-9.

14. Young D, Harrison DA, Cuthbertson BH, Rowan K; TracMan Collaborators. Effect of early vs late tracheostomy placement on survival in patients receiving mechanical ventilation: the TracMan randomized trial. JAMA 2013;309:2121-9.

15. Zagli G, Linden M, Spina R, Bonizzoli M, Cianchi G, Anichini V, et al. Early tracheostomy in intensive care unit: a retrospective study of 506 cases of video-guided Ciaglia Blue Rhino tracheostomies. J Trauma 2010;68:367-72.

16. Durbin CG. Techniques for Performing Tracheostomy. Respir Care 2005;50:488-96.

17. Polderman KH, Spijkstra JJ, deBree R, Christiaans HM, Gelissen HP, Wester JP, Girbes AR. Percutaneous Dilatational Tracheostomy in the ICU: Optimal Organization, Low Complication Rates and a description of a new complication. Chest 2003;123:1595-602.

18. Kost KM. Endoscopic Percutaneous Dilatational Tracheotomy: A prospective Evaluation of 500 Consecutive Cases. Laryngoscope 2005;115:1-30.

19. Rodríguez SJ, Esteves LE. Real-time ultrasound-guided percutaneous dilatational tracheostomy. Crit Care 2011;15:443.

20. Flint AC, Midde R, Rao VA, Lasman TE, Ho PT. Bedside ultrasound screening for pretracheal vascular structures may minimize the risks of percutaneous dilatational tracheostomy. Neurocrit Care 2009;11:372-6

21. Rajajee V, Fletcher JJ, Rochlen LR, Jacobs TL. Real-time ultrasoundguided percutaneous dilatational tracheostomy:a feasibility study. Crit Care 2011;15:R67. 